Pramāna, Vol. 10, No. 3, March 1978, pp. 227-238, (C) Printed in India.

\title{
New light on the optical equivalence theorem and a new type of discrete diagonal coherent state representation $\dagger$
}

\author{
N MUKUNDA and E C G SUDARSHAN* \\ Centre for Theoretical Studies, Indian Institute of Science, Bangalore 560012 \\ *Permanent address: Department of Physics, University of Texas, Austin, Texas, USA \\ MS recevied 10 December 1977
}

\begin{abstract}
In many instances we find it advantageous to display a quantum optical density matrix as a generalized statistical ensemble of coherent wave fields. The weight functions involved in these constructions turn out to belong to a family of distributions, not always smooth functions. In this paper we investigate this question anew and show how it is related to the problem of expanding an arbitrary state in terms of an overcomplete subfamily of the overcomplete set of coherent states. This provides a relatively transparent derivation of the optical equivalence theorem. An interesting by-product is the discovery of a new class of discrete diagonal representations.
\end{abstract}

Keywords. Optical equivalence theorem; coherent states; overcomplete family of states; discrete diagonal representation.

\section{Introduction}

The play of light has been of great interest in physics. The discovery that light has an essential quantum mechanical nature does imply that we have to be careful in the rendering of its behaviour in familiar classical terms. For the most part the phenomena of optics dealt with photometry and other methods of the study of intensity of light. In wave optical processes, the intensity of light on one surface is not fully determined by the intensity of light on an 'earlier' surface: the two-slit interference pattern is the most familiar example in this context. Within the context of wave optics, one is led to introduce the notion of degree of coherence which is then subsumed under the notion of the two-point correlation function. This function is additive for incoherent beams and thus provides a generalization of the notion of intensity; yet in terms of the two-point correlation function, the general propagation can be described. As far as conventional optics is concerned, a field of illumination may be described as a statistical ensemble of wavefields; but how general is such a description of a field of illumination when the full quantum nature of light is included? Remarkably enough a general field of illumination may be described by a classical statistical ensemble as far as the two-point correlation function is concerned (Sudarshan 1969).

There are, however, measurements that can be carried out in an optical field which reflect the full quantum character of light. Photocounting, intensity interferometry,

†Work supported in part by the Energy Research and Development Administration, Contract No. E(40-I)3992. 
and non-linear optics are examples of such measurements. The question naturally arises as to the extent to which an ensemble description in terms of statistical wave fields appropriate to this general context is possible. The diagonal coherent state representation of quantum mechanical density operators (Sudarshan 1963) addresses itself to this question.

The essential characteristic difference of quantum physics from classical statistical physics is the existence of off-diagonal correlation terms which reflect the phasedefinite superposition of quantum states. The success of any attempt to produce a classical ensemble picture of a quantum optical field would depend upon the method of eliminating or transforming away such 'off-diagonal' terms. This is not to be confused with classical limits and classical approximations; on the contrary, the effort is to produce an exact picture. This paper is concerned with the study of this problem and the discovery of a new family of representations.

The diagonal coherent state representation of quantum mechanical density operators is of great heuristic as well as practical value in the quantum description of optical phenomena (Sudarshan 1963). It enables one to express the correlation functions of quantum optics in a form remarkably similar to classical correlation functions defined as averages over classical statistical ensembles. This happens because of two reasons. First, the former correlation functions are defined as expectation values of normal-ordered operator functions of the quantized electromagnetic field. Second, the diagonal representation asserts that any density operator $\rho$ for a system with one degree of freedom is expressible in the form

$$
\rho=\frac{1}{\pi} \int \phi(z)|z\rangle\langle z| d^{2} z
$$

Here the vectors $|z\rangle$ are eigenvectors of the annihilation operator for complex eigenvalues $z$, the coherent states, $\phi$ is a $c$-number weight function, and the integration is over the entire complex plane. The application to quantum optics involves generalising this representation to systems with several degrees of freedom, but for simplicity we restrict ourselves to the case of one degree of freedom.

The two related problems of understanding the nature of the weights $\phi$ that occur in the diagonal representation, and of computing $\phi$ for a given $\rho$, have been extensively discussed in the literature (Mehta and Sudarshan 1965; Klauder et al 1965; Kano 1965; Klauder 1966; Rocca 1966). It is known that $\phi$ is, in general, a distribution. The Fourier transform of $\phi$ is always a distribution on the test function space $D_{2}$ (space of functions of two real variables which are infinitely often differentiable and have compact support), so that $\phi$ itself is a distribution on the space $Z_{2}$ obtained by applying the Fourier transformation to $D_{2}$. Furthermore, it has been shown that a given $\rho$ can be approximated to arbitrary accuracy in the trace-class norm by a sequence of density operators whose associated weight functions are infinitely often differentiable and vanish at infinity faster than any inverse powers of their arguments. (See for instance Klauder and Sudarshan 1968). As for computing $\phi$ for a given $\rho$, two methods have generally been adopted. The first uses the diagonal coherent state matrix elements of $\rho,\langle z|\rho| z\rangle$ for all $z$, for the construction of $\phi$, since it is known that these matrix elements do specify $\rho$ uniquely. In the second method, one uses instead the antidiagonal coherent state matrix elements $\langle-z|\rho| z\rangle$, 
since that leads to a simpler equation for the determination of $\phi$ (Mehta 1967, Agarwal and Wolf 1970).

It is clear that the use of coherent states and the diagonal representation involves analysis at two distinct levels, one at the level of vectors in the Hilbert space to which the coherent states themselves belong, and another at the level of operators on this Hilbert space. The existence and essential properties of the coherent states are directly related to one's having a solution of the canonical Heisenberg commutation relation at hand. One of the aims of this paper is to show that the discussion relating to operators has exactly the same underlying mathematical structure as the discussion relating to state vectors, because the operators can be viewed as a linear space supporting a solution of Heisenberg commutation relations for two degrees of freedom. As a result, many of the questions that come up at the operator level have exact parallels at a simpler level. This is in particular true of the attempt to set up the diagonal representation, and of the two common ways of determining $\phi$ for a given $\rho$. The second aim of this paper is to exploit this relationship and show that any density operator $\rho$ can be approximated arbitrarily closely by discrete diagonal coherent state representations in which, essentially, the weight $\phi$ is concentrated on certain countable infinite sets in the complex plane. This remarkable possibility occurs because while the coherent states form an overcomplete set, certain countable subsets of them form complete sets in Hilbert space.

The material of this paper is arranged as follows. In section 2 we recall briefly those essential properties of coherent states that will be needed later. Section 3 sets up the Weyl representation of operators on a Hilbert space and the two alternative methods of calculating the weight $\phi$ in the diagonal representation. These two sections are intended mainly to settle notation and make this paper self-contained. In section 4 we analyze the mathematical structure underlying the diagonal representation and show its complete equivalence to the structure used in the description of state vectors in terms of coherent states. This equivalence is then used in section 5 to prove the existence of new, discrete forms of the diagonal representation. Concluding remarks and questions comprise section 6 .

\section{Basic properties of coherent states}

The canonical Heisenberg commutation relation, which underlies the construction of coherent states, involves two hermitian operators $q, p$ obeying

$$
[q, p]=i \text {. }
$$

The unitary Weyl form of this relation is expressed in terms of two one-parameter families of operators $U(\sigma), V(\tau)$ defined by

$$
U(\sigma)=\exp (i \sigma q), V(\tau)=\exp (i \tau p),-\infty<\sigma, \tau<\infty .
$$

They obey

$$
\begin{gathered}
U(\sigma) U\left(\sigma^{\prime}\right)=U\left(\sigma+\sigma^{\prime}\right), V(\tau) V\left(\tau^{\prime}\right)=V\left(\tau+\tau^{\prime}\right), \\
U(\sigma) V(\tau)=V(\tau) U(\sigma) \exp (-i c \tau)
\end{gathered}
$$


The annihilation operator $a$ and its adjoint $a^{\dagger}$ are defined as

$$
a=(q+i p) / \sqrt{2}, a^{\dagger}=(q-i p) / \sqrt{2}
$$

and then eq. (1) becomes

$$
\left[a, a^{\dagger}\right]=1
$$

Coherent states are eigenvectors of the annihilation operator. There is one eigenvector $|z\rangle$ corresponding to each complex number $z$ as eigenvalue:

$$
a|z\rangle=z|z\rangle \text {. }
$$

The Schrödinger wavefunctions of these eigenvectors are

$$
\left\langle q^{\prime} \mid z\right\rangle=\pi^{-1} \exp \left[-\frac{1}{2}\left(q^{\prime}-z \sqrt{ } 2\right)^{2}-\frac{1}{2} z\left(z^{*}-z\right)\right] .
$$

Here $q^{\prime}$ is a general eigenvalue of the operator $q$ and the corresponding eigenvectors $\left|q^{\prime}\right\rangle$ are subject to the usual delta function normalization. Coherent states form a non-orthonormal set since

$$
\left\langle z^{\prime} \mid z\right\rangle=\exp \left(-\frac{1}{2}\left|z^{\prime}\right|^{2}-\frac{1}{2}|z|^{2}+z^{\prime *} z\right)
$$

A general vector $|\psi\rangle$ is describable by its Schrödinger wave function $\psi\left(q^{\prime}\right)$ which is a square integrable function of $q^{\prime}$. It can also be described by its projections on all coherent states. If one writes

$$
\left\langle z^{*} \mid \psi\right\rangle==\exp \left(-\frac{1}{2}|z|^{2}\right) f(z),
$$

then $f(z)$ is entire analytic and obeys a growth condition at infinity:

$$
|f(z)| \leqslant \||\psi\rangle \| \exp \left(\frac{1}{2}|z|^{2}\right) .
$$

Using eq. (7) we can relate $f(z)$ to $\psi\left(q^{\prime}\right)$ :

$$
f(z)=\pi^{-1 / 4} \exp \left(-\frac{1}{2} z^{2}\right) \int_{-\infty}^{\infty} \psi\left(q^{\prime}\right) \exp \left(-\frac{1}{2} q^{\prime 2}+q^{\prime} z \sqrt{2}\right) d q^{\prime}
$$

The coherent states $|z\rangle$ taken for all $z$ form an overcomplete set. One has a resolution of the identity expressed by the following equation valid for any $|\psi\rangle$ (Klauder 1960):

$$
|\psi\rangle=\pi^{-1} \int|z\rangle\langle z \mid \psi\rangle d^{2} z
$$

Certain subsets of the coherent states form complete sets in Hilbert space; the precise meaning of this and some examples will be given in section 5, though some cases will be used in section 4 as well. The complete equivalence of the Schrödinger description 
of the Hilbert space using square integrable wave functions $\psi\left(q^{\prime}\right)$ and the description using entire analytic functions $f(z)$, based on eq. (9), has been established by Bargmann (Bargmann 1961).

\section{The diagonal representation}

As stated in the introduction, the properties of the diagonal representation have been studied in the literature in the context of the trace-class norm for operators. Our discussion however will be based on the Hilbert-Schmidt norm. Let $\mathscr{H}$ denote the Hilbert space to which the discussion of the previous section refers. A linear operator $A$ on $\mathscr{H}$ is of Hilbert-Schmidt $(\mathrm{H}-\mathrm{S})$ class if

$$
\operatorname{Tr}\left(A^{\dagger} A\right)<\infty
$$

The collection of all such operators forms a linear set and in fact a Hilbert space, $\mathscr{K}$ say, if we define the inner product of two operators by

$$
(A, B)=\operatorname{Tr}\left(A^{\dagger} B\right)
$$

The density operators not only belong to the trace-class but also to the HilbertSchmidt class. So the density operators and their representations are automatically included in the present study. Analogous to the Schrödinger wave function description of vectors in $\mathscr{H}$, one has a Weyl representation for the elements of $\mathscr{K}$ (Weyl 1931). The basic operators used here are the so-called displacement operators $D(a)$ :

$$
D(a)=\pi^{-1 / 2} \exp \left(a a^{\dagger}-a^{*} a\right)
$$

$a$ is a complex number, and apart from the factor $\pi^{-1 / 2}, D(a)$ is unitary. These operators are improper elements of $\mathscr{K}$, like eigenvectors of $q$ in $\mathscr{H}$, and they obey

$$
\left(D\left(a^{\prime}\right), D(a)\right)=\delta\left(a^{\prime}-a\right) \equiv \delta\left(\operatorname{Re} a^{\prime}-\operatorname{Re} a\right) \delta\left(\operatorname{Im} a^{\prime}-\operatorname{Im} a\right)
$$

The Weyl representation expands a general H-S operator $T$ in terms of the displacement operators:

$$
\begin{gathered}
T=\int t(a) D(a) d^{2} a, t(a)=(D(a), T), \\
(T, T)=\int|t(a)|^{2} d^{2} a
\end{gathered}
$$

Thus elements of $\mathscr{K}$ correspond one-to-one to square-integrable functions of two real variables, their Weyl weights. (For a rigorous discussion, see Pool 1966). The diagonal coherent state matrix elements of $D(a)$ are needed later and are

$$
\langle z|D(a)| z\rangle=\pi^{-1 / 2} \exp \left(-\frac{1}{2}|a|^{2}+a z^{*}-a^{*} z\right) .
$$


Let $\phi$ be the weight function appearing in the diagonal representation of an element $T$ in $\mathscr{K}:$

$$
T=\frac{1}{\pi} \int \phi(z)|z\rangle\langle z| d^{2} z
$$

One way to find $\phi$ is to take the diagonal coherent state matrix element of this relation. It turns out that then the Fourier transform of $\phi$ gets determined in terms of the Weyl weight of $T$. From eqs (8), (17), (18) and (19) we get:

$$
\begin{aligned}
\int t(\alpha) \exp & \left(-\frac{1}{2}|a|^{2}+a z^{* *}-a^{*} z^{\prime}\right) d^{2} \alpha \\
= & \pi^{-1 / 2} \int \phi(z) \exp \left(-\left|z-z^{\prime}\right|^{2}\right) d^{2} z
\end{aligned}
$$

If $\tilde{\phi}$ is the Fourier transform of $\phi$ :

$$
\phi(z)=\frac{1}{\pi} \int \tilde{\phi}(\alpha) \exp \left(a z^{*}-\alpha^{*} z\right) d^{2} a
$$

then from eq. (20) we get the result

$$
\tilde{\phi}(a)=\pi^{1 / 2} t(a) \exp \left(\frac{1}{2}|a|^{2}\right)
$$

Since $t(a)$ is square-integrable, it is the exponential factor here that forces one to interpret $\tilde{\phi}$, and so $\phi$ as well, as a distribution.

The alternative method of finding $\phi$ uses the antidiagonal coherent state matrix elements of eq. (19). When eq. (8) is used, this reads:

$$
\begin{aligned}
& \left\langle-z^{\prime}|T| z^{\prime}\right\rangle= \\
& \quad \frac{1}{\pi} \int \phi(z) \exp \left(-|z|^{2}-\left|z^{\prime}\right|^{2}+z^{*} z^{\prime}-z z^{\prime *}\right) d^{2} z .
\end{aligned}
$$

The right hand side has the form of a Fourier transformation which can be inverted to yield $\phi$ :

$$
\begin{aligned}
\phi(z)=(1 / \pi) & \exp \left(|z|^{2}\right) \int\left\langle-z^{\prime}|T| z^{\prime}\right\rangle \\
& \exp \left(\left|z^{\prime}\right|^{2}+z z^{\prime *}-z^{*} z^{\prime}\right) d^{2} z^{\prime} .
\end{aligned}
$$

An alternative derivation of eq. (22) is obtained by taking the expectation value of both sides of eq. (17) using eq. (18) to obtain

$$
\pi^{-1 / 2} \int t(a) \exp \left(-\frac{1}{2}|a|^{2}+a z^{*}-a^{*} z\right) d^{2} a=\langle z|T| z\rangle .
$$

Taking Fourier inverse we get

$$
t(a)=\pi^{-1 / 2} \exp \left(\frac{1}{2}|a|^{2}\right) \int\langle z|T| z\rangle \exp \left(a^{*} z-a z^{*}\right) d^{2} z .
$$


Since the Fourier transform of $\phi(z)$ is $\exp \left(|a|^{2}\right)$ times the Fourier transform of $\langle z|T| z\rangle$ (Mehta and Sudarshan 1965), eq. (22) follows.

\section{Reinterpretation of the diagonal representation}

In this section we intend to show that the mathematical structure underlying the diagonal representation is identical in essential respects to the structure present at the level of the state vector space $\mathscr{H}$. To do this we first show that, using the Weyl system of operators $U(\sigma), V(\tau)$ of eq. (2) acting on $\mathcal{H}$, we can in a natural way set up two commuting Weyl systems acting on $\mathscr{K}$. With $A$ denoting a general element of $\mathscr{K}$, we define operators $\mathscr{U}_{j}(\sigma), \mathscr{V}_{j}(\tau), j=1,2$, on $\mathscr{K}$ thus:

$$
\begin{aligned}
& \varkappa_{1}(\sigma) A=U(\sigma / \sqrt{2}) A U(-\sigma / \sqrt{2}), \quad U_{2}(\sigma) A=V(\sigma / \sqrt{2}) A V(-\sigma / \sqrt{2}), \\
& \mathscr{V}_{1}(\tau) A=V(\tau / \sqrt{2}) A V(\tau / \sqrt{2}), \quad \mathscr{V}_{2}(\tau) A=U(-\tau / \sqrt{2}) A U(-\tau / \sqrt{2})
\end{aligned}
$$

It is straightforward to check first that these linear operators on $\mathscr{K}$ do preserve the inner product (14), and to verify next using eq. (3) that $\mathscr{U}_{1}(\sigma), \mathscr{V}_{1}(\tau)$ and $\mathscr{U}_{2}(\sigma)$, $\mathscr{V}_{2}(\tau)$ form two mutually commuting Weyl systems. That is, one has

$$
\begin{aligned}
& \mathscr{U}_{1}(\sigma) \mathscr{U}_{1}\left(\sigma^{\prime}\right)=\mathscr{U}_{1}\left(\sigma+\sigma^{\prime}\right), \mathscr{V}_{1}(\tau) \mathscr{V}_{1}\left(\tau^{\prime}\right)=\mathscr{V}_{1}\left(\tau+\tau^{\prime}\right), \\
& \mathcal{U}_{1}(\sigma) \mathscr{V}_{1}(\tau)=\mathscr{V}_{1}(\tau) \mathscr{U}_{1}(\sigma) \exp (-i \sigma \tau),
\end{aligned}
$$

and exactly similar equations for $\mathscr{U}_{2}(\sigma), \mathscr{V}_{2}(\tau)$; and operators with subscript 1 commute with those with subscript 2 . The infinitesimal generators of the one-parameter families defined in eq. (25) are found to be

$$
\begin{aligned}
& Q_{1} A=[q, A] / \sqrt{2}, Q_{2} A=[p, A] / \sqrt{2}, \\
& P_{1} A=\{p, A\} / \sqrt{2}, P_{2} A=-\{q, A\} / \sqrt{2} .
\end{aligned}
$$

The operators $Q_{j}, P_{j}$ are hermitian with respect to the inner product on $\mathscr{K}$, and obey Heisenberg commutation relations

$$
\left[Q_{J}, Q_{k}\right]=\left[P_{J}, P_{k}\right]=0,\left[Q_{J}, P_{k}\right]=i \delta_{j k}
$$

It is now natural to ask for a Schrödinger type description of the space $\mathscr{K}$, which diagonalises $Q_{j}$ while the $P_{j}$ are operators of partial differentiation. One finds that the Weyl representation (17) does exactly this. The displacement operators $D(\alpha)$ of eq (15) are in fact simultaneous ' eigenvectors' of $Q_{1}$ and $Q_{2}$. Writing $a=r+i s$ with $r$ and $s$ real, one finds

$$
\begin{aligned}
& Q_{1} D(a)=r D(a), \quad Q_{2} D(a)=s D(a) \\
& P_{1} D(a)=i \frac{\partial}{\partial r} D(a), \quad P_{2} D(a)=i \frac{\partial}{\partial s} D(\alpha)
\end{aligned}
$$


Therefore, the association of the Weyl weight $t(\alpha)$ with an element $T$ in $\mathscr{K}$ is exactly similar to the association of a Schrödinger wave function $\psi\left(q^{\prime}\right)$ with a vector $|\psi\rangle$ in $\mathscr{H}$.

The next natural step is to carry over the description of $\mathscr{H}$ using coherent states, to the level of $\mathscr{K}$. We now have two independent annihilation operators $\mathscr{A}_{1}, \mathscr{A}_{2}$ and their adjoints, defined in terms of $Q_{J}$ and $P_{J}$ in the manner of eq (4):

$$
\begin{aligned}
& \mathscr{A}_{1} A=\left(a A-A a^{\dagger}\right) / \sqrt{2}, \mathscr{A}_{2} A=-i\left(a A+A a^{\dagger}\right) / \sqrt{2}, \\
& \mathscr{A}_{1}^{\dagger} A=\left(a^{\dagger} A-A a\right) / \sqrt{2}, \mathscr{A}_{2}^{\dagger} A=i\left(a^{\dagger} A+A a\right) / \sqrt{2} \overline{-}
\end{aligned}
$$

The simultaneous 'eigenvectors' of $\mathscr{A}_{1}$ and $\mathscr{A}_{2}$ are those elements of $\mathscr{K}$ that are formed from coherent states in $\mathscr{H}$ and their adjoints as outer products:

$$
\begin{aligned}
& \mathscr{A}_{1}\left|z_{1}\right\rangle\left\langle z_{2}\left|=\frac{\left(z_{1}-z_{2}^{*}\right)}{\sqrt{2}}\right| z_{1}\right\rangle\left\langle z_{2}\right|, \\
& \mathscr{A}_{2}\left|z_{1}\right\rangle\left\langle z_{2}\left|=-i \frac{\left(z_{1}+z_{2}^{*}\right)}{\sqrt{2}}\right| z_{1}\right\rangle\left\langle z_{2}\right| .
\end{aligned}
$$

One finds that these elements of $\mathscr{K}$ have inner products with one another that are a direct generalization of eq. (8) to two degrees of freedom:

$$
\begin{aligned}
\left(\left|z_{3}\right\rangle\left\langle z_{4}|,| z_{1}\right\rangle\left\langle z_{2}\right|\right) \equiv\left\langle z_{3} \mid z_{1}\right\rangle\left\langle z_{2} \mid z_{4}\right\rangle \\
=\exp \left(-\frac{1}{4}\left|z_{1}-z_{2}{ }^{*}\right|^{2}-\frac{1}{4}\left|z_{3}-z_{4}{ }^{*}\right|^{2}+\frac{1}{2}\left(z_{3}^{*}-z_{4}\right)\left(z_{1}-z_{2}{ }^{*}\right)\right. \\
\left.\quad-\frac{1}{4}\left|z_{1}+z_{2}{ }^{*}\right|^{2}-\frac{1}{4}\left|z_{3}+z_{4}{ }^{*}\right|^{2}+\frac{1}{2}\left(z_{3}{ }^{*}+z_{4}\right)\left(z_{1}+z_{2}{ }^{*}\right)\right) .
\end{aligned}
$$

The result that $f(z)$ in eq. (9) is entire analytic, and the resolution of the identity in eq. (12), both generalise easily to $\mathscr{K}$. (For the former, see Mehta and Sudarshan 1965).

In the light of the above construction, the diagonal coherent state representation (19) finds a new interpretation. The elements of $\mathscr{K}$ used in it are 'eigenvectors' of $\mathscr{A}_{1}$ and $\mathscr{A}_{2}$ with pure imaginary eigenvalues for both:

$$
\begin{aligned}
& \mathscr{A}_{1}|z\rangle\langle z|=(i \sqrt{2} \operatorname{Im} z)| z\rangle\langle z|, \\
& \mathscr{A}_{2}|z\rangle\langle z|=(-i \sqrt{2} \operatorname{Re} z)| z\rangle\langle z| .
\end{aligned}
$$

Therefore the problem of setting up the diagonal representation for operators on $\mathscr{H}$ is exactly the same as that of replacing the expansion (12) for a vector $|\psi\rangle$ in $\mathscr{H}$ with one in which only coherent states with pure imaginary eigenvalues of $a$ are used. Such an expansion for $|\psi\rangle$ has the form

$$
|\psi\rangle=\pi^{-\frac{1}{2}} \int_{-\infty}^{\infty} v(r)|i \sqrt{2} r\rangle d r .
$$

One can hope that this representation for any $|\psi\rangle$ is possible because the subset of coherent states appearing here is complete (actually overcomplete-see the next 
section). The need to permit the occurrence of distributions as weight functions $\phi$ in eq. (19) arises from a corresponding need to permit distributions to appear as 'wave functions' $v(r)$ in the simpler situation presented by eq. (34).

The analogue, at the vector level, of the first method of obtaining $\phi(z)$ is the following. We take the inner product of both sides of eq. (34) with a coherent state $|i \sqrt{2} s\rangle$ where $s$ is real, and then use eqs (7) and (8):

$$
\begin{aligned}
& \int_{-\infty}^{\infty} \psi\left(q^{\prime}\right) \exp \left(-\frac{1}{2} q^{\prime 2}+2 i s q^{\prime}\right) d q^{\prime} \\
& \quad=\pi^{-1 / 4} \int_{-\infty}^{\infty} v(r) \exp \left[-(r-s)^{2}\right] d r
\end{aligned}
$$

This corresponds to eq. (20); we then follow eq. (21) and set up a Fourier representation for $v(r)$

$$
v(r)=\pi^{-1 / 2} \int_{-\infty}^{\infty} \tilde{v}\left(q^{\prime}\right) \exp \left(2 i r q^{\prime}\right) d q^{\prime}
$$

On combining eqs (35) and (36) we get

$$
\widetilde{v}\left(q^{\prime}\right)=\pi^{1 / 4} \psi\left(q^{\prime}\right) \exp \left(\frac{1}{2} q^{\prime 2}\right)
$$

which corresponds exactly to eq. (22) since the Schrödinger wave function and the Weyl weight are analogous quantities. In view of the last factor in eq. (37), the inverse Fourier transform $v(r)$ and hence the representation (34) may involve distributions rather than functions. Unlike the overcomplete family of states $|z\rangle$ with $z$ complex, the family $|i \sqrt{2} r\rangle$ with $r$ real requires possibly more singular combinations.

As for the second method for finding $\phi(z)$, we realize that the left hand side of eq. (23) can be written as

$$
\left\langle-z^{\prime}|T| z^{\prime}\right\rangle=\left(\left|-z^{\prime}\right\rangle\left\langle z^{\prime}\right|, T\right)
$$

and that the eigenvector of $\mathscr{A}_{1}$ and $\mathscr{A}_{2}$ occurring here corresponds to real eigenvalues for both. The analogous procedure to determine $v(r)$ at the state vector level is then to take the inner product of both sides of eq. (34) with a coherent state $|\sqrt{2} s\rangle$ where $s$ is real:

$$
\langle\sqrt{2} s \mid \psi\rangle=\pi^{-1 / 2} \int_{-\infty}^{\infty} v(r) \exp \left(-s^{2}-r^{2}+2 i r s\right) d r
$$

This corresponds to eq. (23). Inversion of the Fourier transform then gives the analogue to eq. (24):

$$
v(r)=\pi^{-1 / 2} \exp \left(r^{2}\right) \int_{-\infty}^{\infty}\langle\sqrt{2} s \mid \psi\rangle \exp \left(s^{2}-2 i r s\right) d s
$$

This discussion shows that, at least for H-S operators, the diagonal representation and questions connected with it are two-dimensional versions of simpler questions at a one-dimensional level. 


\section{Discrete diagonal representations}

The fact that the coherent states, and even the subsets of states $|i \sqrt{2} r\rangle$ or $|\sqrt{2} s\rangle$ with $r, s$ real are overcomplete implies that not every value of the weight function in expanding or approximating an arbitrary state $|\psi\rangle$ contains 'information'. It would be interesting to know what 'information' is contained in arbitrary statistical states. To evaluate the information content we should get the state in a classical like ensemble with no non-diagonal terms and, that, in terms of the fewest number of states. Clearly the state should be overcomplete: otherwise we could not get a diagonal form for every statistical state expanded with the same set of states. We should thus reconcile two opposing tendencies.

We now use the results of the last section to exhibit new forms of the diagonal representation. For this, recall from section 2 that every vector $|\psi\rangle$ stands in one-one correspondence with an entire analytic function $f(z)$ according to eq. (9). We now say a subset $S$ of the complex plane is a characteristic set if the vanishing of $f$ at all points of $S$ implies the identical vanishing of $f$. Equivalently, $S$ is a characteristic set if

$$
\langle z \mid \psi\rangle=0 \text {, all } z \in S \Longrightarrow|\psi\rangle=0
$$

(Bargmann 1961). Examples of such sets that do not specifically refer to the large $z$ behaviour of $f$, eq (10), are: (1) any set of points in the complex plane with a finite limit point; (ii) the set of all real numbers; (iii) the set of all imaginary numbers, etc. (Sets (ii) and (iii) were used in the previous section in reconstructing $|\psi\rangle$ from $\langle i \sqrt{2} s \mid \psi\rangle$ or $\langle\sqrt{2} s \mid \psi\rangle$ for all real $s$ ). Examples of characteristic sets that do reflect the large $z$ behaviour of $f(z)$ are (Bargmann 1961): any sequence $\left\{a_{n}\right\}$ of distinct nonzero complex numbers such that

$$
\sum_{1}^{\infty}\left|a_{n}\right|^{-2-n}=\infty
$$

for some $\eta>0$.

For simplicity, we consider only countable characteristic sets. Let the points of $S$ be $z_{n}, n=1,2, \ldots$ Then the set of (distinct) coherent states $\left|z_{n}\right\rangle$ has the property

$$
\left\langle z_{n} \mid \psi\right\rangle=0, \text { all } n \Longrightarrow|\psi\rangle=0
$$

This implies that the set of states $\left\{\left|z_{n}\right\rangle\right\}$ is complete in the following precise sense: (i) every finite subset of this set is linearly independent; (ii) the linear manifold of all finite linear combinations of vectors drawn from $\left\{\left|z_{n}\right\rangle\right\}$ is dense in $\mathscr{H}$. In detail, (ii) means: a given vector $|\psi\rangle$ in $\mathscr{H}$ can be approximated to any desired accuracy by a suitable finite linear combination of vectors $\left|z_{n}\right\rangle$. So, given any $\epsilon>0$, one can find an integer $N(\epsilon)<\infty$ and coefficients $a_{n}(\epsilon)$ such that

$$
\||\psi\rangle-\sum_{1}^{N(\epsilon)} a_{n}(\epsilon)\left|z_{n}\right\rangle \|<\epsilon
$$

However, because the $\left|z_{n}\right\rangle$ do not form an orthonormal set, their completeness does 
not imply the existence of a definite set of expansion coefficients $a_{n}$ for a given $|\psi\rangle$ such that the finite sums

$$
\sum_{1}^{N} a_{n}\left|z_{n}\right\rangle
$$

converge to $|\psi\rangle$ in the norm as $N \rightarrow \infty$. In other words, with a nonorthonormal complete set, it is in general not possible to 'actually' write a given vector $|\psi\rangle$ as a linear combination of the vectors in the set, though with finite linear combinations one can get as close to $|\psi\rangle$ as one wishes. Essentially this same problem occurs in the subject of nonharmonic Fourier series (Paley and Wiener 1934, Levinson 1940).

The discrete diagonal representations now arise as follows. Take two characteristic sets $S_{1}$ and $S_{2}$, both countable and both consisting of points on the imaginary axis only. Let them consist of the points $\left\{i \sqrt{2} y_{n}\right\}$ and $\left\{-i \sqrt{2} x_{n}\right\}$ respectively, with $x_{n}$ and $y_{n}$ real. It follows that the set of simultaneous 'eigenvectors' of $\mathscr{A}_{1}$ and $\mathscr{A}_{2}$ corresponding to the respective eigenvalues $i \sqrt{2} y_{n}$ and $-i \sqrt{2} x_{m}$ is complete in $\mathscr{K}$ in the precise sense defined above. Here $m$ and $n$ go independently from 1 to $\infty$. Comparison with eq (33) shows immediately that this set of elements in $\mathscr{K}$ is

$$
\left|z_{m n}\right\rangle\left\langle z_{m n}\right|, z_{m n}=x_{m}+i y_{n}, m, n,=1,2, \ldots
$$

This again is countable. We conclude therefore that any density operator $\rho$ can be approximated to arbitrary accuracy in the H-S norm by finite discrete diagonal coherent state expressions of the form.

$$
\sum_{m, n} \phi_{m n}\left|z_{m n}\right\rangle\left\langle z_{m n}\right|
$$

where $\left\{z_{m n}\right\}$ is a preassigned countable set of complex numbers built up as described above. Such approximations to $\rho$ share with the usual diagonal representation (19) the property that when quantum correlation functions are calculated using them, one gets classical-looking forms. But the main difference is that in general there is no definite sequence of expansion coefficients $\left\{\phi_{m n}\right\}$ associated with a given $\rho$, whereas there is a definite weight function $\phi(z)$, albeit a distribution.

\section{Concluding remarks}

In this paper we have presented a new way of understanding the structure of the diagonal representation in quantum optics. When the state vector space corresponds to a system with one degree of freedom, the representation of operators is most naturally discussed in the language appropriate to a system with two degrees of freedom. This makes the form of the diagonal representation as well as its explicit determination very transparent.

We have also seen some of the subtle consequences of the overcompleteness property of coherent states. The most striking is that one can replace the usual continuous form of the diagonal representation (19) by the discrete form (46). At first sight this seems to present a paradox because the weight function $\phi(z)$ of eq. (19) does appear to 
be unambiguously determined by the given operator $T$. One sees no room for all the freedom present in representations of the form (46), which naively speaking arise from a $\phi(z)$ which is a sum of delta functions concentrated at a given sequence of points $\left\{z_{m n}\right\}$ in the complex plane. But the paradox is avoided by the subtle distincttion between the completeness of a given sequence of coherent states, and the possibility of actually expanding a general vector in terms of these states. It is of interest to examine this question more closely and find out which characteristic sets produce complete sets of coherent states that also possess the expansion possibility.

The algebraic approach of this paper led naturally to the Hilbert-Schmidt norm for operators on the Hilbert space $\mathscr{H}$, and the convergence of various operator representations is understood to be in the sense of this norm. However, for applications one would prefer that any sequence of operators converging to a given density operator $\rho$ do so in the trace-class norm, so that the sequence can be used for calculating expectation values. The existing proofs of the diagonal representation, with $\phi(z)$ a distribution, do work with the trace-class norm (Klauder and Sudarshan 1968). It would be interesting to extend the methods of this paper in that direction. We hope to examine these questions elsewhere.

\section{Acknowledgement}

It is a pleasure to thank Profs $\mathrm{K}$ Balagangadharan and $\mathrm{J}$ Pasupathy for many useful discussions.

\section{References}

Agarwal G S and Wolf E 1970 Phys. Rev. D2 2161

Bargmann V 1961 Commun. Pure Appl. Math. 14187

Kano Y 1965 J. Math. Phys. 61913

Klauder J R 1960 Ann. Phys. (N.Y) 11123

Klauder J R, McKenna J and Currie D G 1965 J. Math. Phys. 6733

Klauder J R 1966 Phys. Rev. Lett. 16534

Klauder J R and Sudarshan E C G 1968 Fundamentals of Quuntum Optics (New York: W A Benjamin), Ch. 8

Levinson N 1940 Gap and Density Theorems AMS Colloquium Pub. Vol. 26, Ch. IV

Mehta C L and Sudarshan E C G 1965 Phys. Rev. 138B 274

Mehta C L 1967 Phys. Rev. Lett. 18752

Paley R E A C and Wiener N 1934 Fourier Transforms in the Complex Domain AMS Colloquium Publications Vol. 19, Ch. VI, VII

Pool J C T 1966 J. Math. Phys. 766

Rocca F 1966 Compt. Rend. 262 A547

Sudarshan E C G 1963 Phys. Rev. Lett. 10277

Sudarshan E C G 1969 J. Math. Phys. Sci. 3121

Weyl H 1931 The Theory of Groups and Quantum Mechanics (New York: Dover), p. 274 